Produto \& Produção, vol. 18, n.2, p.13-19. 2017

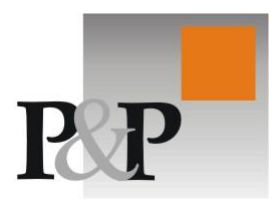

RECEBIDO EM 18/11/2015. ACEITO EM 12/05/2017.

\author{
Caroline Rodrigues Vaz \\ Universidade Federal de Santa Catarina - UFSC \\ karollrvaz@gmail.com
}

Mauricio Uriona Maldonado

Universidade Federal de Santa Catarina - UFSC

\title{
Efeito Chicote em Redes de Logística Reversa: um Modelo de Simulação Dinâmica
}

\section{Resumo}

Enquanto as indústrias de manufatura ingressam na era da sustentabilidade, mudanças dramáticas são evidenciadas nas suas cadeias de suprimento, a mais importante, que além do fluxo tradicional de materiais desde os fornecedores até os consumidores finais, existem também, fluxos reversos de materiais, conhecidos como logística reversa. O objetivo do artigo é visualizar os efeitos de amplificação (efeito chicote) em cadeias de suprimento com logística reversa. Para isto, um modelo de dinâmica de sistemas é desenvolvido, a partir dos dados de uma empresa do setor eletroeletrônico. O modelo serve para identificar os impactos no efeito chicote quando da implantação de cadeias de suprimento com logística reversa. Os resultados apresentam uma piora do efeito chicote quando operações de logística reversa são implantadas na empresa. Apresentam também, um tipo de efeito chicote, do ponto de vista da variação com a cadeia tradicional, quando do afastamento progressivo entre o consumidor final e os elos da cadeia.

Palavras-chave: Logística Reversa, Efeito Chicote, Dinâmica de Sistemas, Cadeias de Suprimento de malha fechada.

\begin{abstract}
While manufacturing industries enter the sustainability age, dramatic changes in their supply chains are evidenced. The most important change is that, besides the forward flow of materials, from suppliers to end customers, companies have now reverse flows of materials, known as reverse logistics. The main objetive of this paper is to visualize the amplification effects (bullwhip effect) in supply chains with reverse logistics networks. In order to achieve this, a system dynamics model is built. The model serves to assess the impact on the bullwhip effect when reverse logistics networks are implemented. The results show an increase in the bullwhip effect when reverse logistics are set up. The results also show a sort of bullwhip effect of the variation between traditional and reverse logistics supply chain, by showing amplification downstream.
\end{abstract}

Key-word: Reverse Logistics, Bullwhip Effect, System Dynamics, Closed-Loop Supply Chains.

\section{Introdução}

A logística reversa apresenta-se como uma estratégia promissora no que tange à redução de desperdiço e resíduos de produtos manufaturados, coadjuvando com o consumo consciente e com a 
redução de gases de efeito estufa, produzidos pelas operações industriais. Ao mesmo tempo, a logística reversa representa uma mudança radical das operações de produção de uma empresa, pois novos atores, variáveis, processos e até legislações devem ser incorporados ao dia-a-dia da mesma, se o que se deseja é ter sucesso com a sua implantação.

Isto significa incrementar a complexidade da gestão da cadeia bem como dos próprios mecanismos de controle e de avaliação de desempenho. Significa também que outros processos ou até efeitos indiretos sejam afetados pela implantação de cadeias de logística reversa, tais como possíveis impactos negativos no efeito chicote - a amplificação da demanda ao longo da cadeia de suprimentos (GEARY; DISNEY; TOWILL, 2006), pelo fato de incorporar novos fluxos materiais e de informação.

Uma forma de avaliar os efeitos críticos relacionados com essa complexidade é través da formulação de modelos de simulação (BORSHCHEV; FILIPPOV, 2004). Por sua vez, uma das metodologias de simulação que tem-se destacado nas últimas décadas no estudo de cadeias de suprimentos é a Dinâmica de Sistemas - DS (System Dynamics - em inglês) (ANGERHOFER; ANGELIDES, 2000) que tem como princípio de funcionamento a identificação de fluxos de realimentação que podem provocar instabilidade na cadeia, bem como as regras de decisão subjacentes a esses fluxos.

Interessantemente, a primeira referência do efeito chicote ou bullwhip effect na literatura é a obra de J. Forrester (FORRESTER, 1961), na qual o autor propunha a primeira versão da DS, como ferramenta de simulação dinâmica baseada nos ciclos de realimentação. Mais tarde, Sterman (1989) relatou e evidenciou o efeito chicote em um jogo de empresas - utilizando DS - denominado "beer game" ou "jogo da cerveja".

Neste sentido, o objetivo do artigo é apresentar o impacto da implantação de cadeias logísticas reversas no efeito chicote das empresas que a implantaram, seguindo a linha de trabalhos similares, porém escassos, como o de Spengler e Schroeter (2003) e Vlachos et al. (2007).

\section{Logística Reversa}

A rede de logística reversa (LR) tem como propósito coletar produtos usados ou devolvidos dos clientes/consumidores, consolidá-los, inspecioná-los e transportá-los de volta para a linha de produção (FERGUSON; SOUZA, 2010) bem como para o descarte apropriado. As redes de logística reversa se configuram como malhas fechadas pois a quantidade de produtos coletados determina a velocidade e magnitude das atividades de reuso, e reciclagem desses materiais em produtos novos ou reusados.

Vários têm sido os autores que oferecem definições da LR, entre eles Stock (1992), Carter e Ellram (1998) e Rogers e Tibben-Lembke (1999) dentre outros, para eles, a logística reversa é o processo de planejamento, implementação e controle eficiente do fluxo de matérias primas, produto em processo, produto acabado e o fluxo de informações, desde o ponto de consumo até o ponto de origem, com o propósito de recuperação de valor ou para o descarte apropriado (ROGERS, DALE S.; TIBBENLEMBKE, 1999) .

Para Stock (1998) a LR é um modelo sistêmico que aplica os melhores métodos da engenharia e da administração logística, com o objetivo de fechar lucrativamente o ciclo da cadeia de suprimentos. A empresa que inicia o processo de Logística Reversa ganha tanto no fornecimento de uma imagem institucional positiva, quanto na visão de responsabilidade empresarial - meio ambiente e sociedade (VAZ et al., 2013), conforme a Figura 1. 


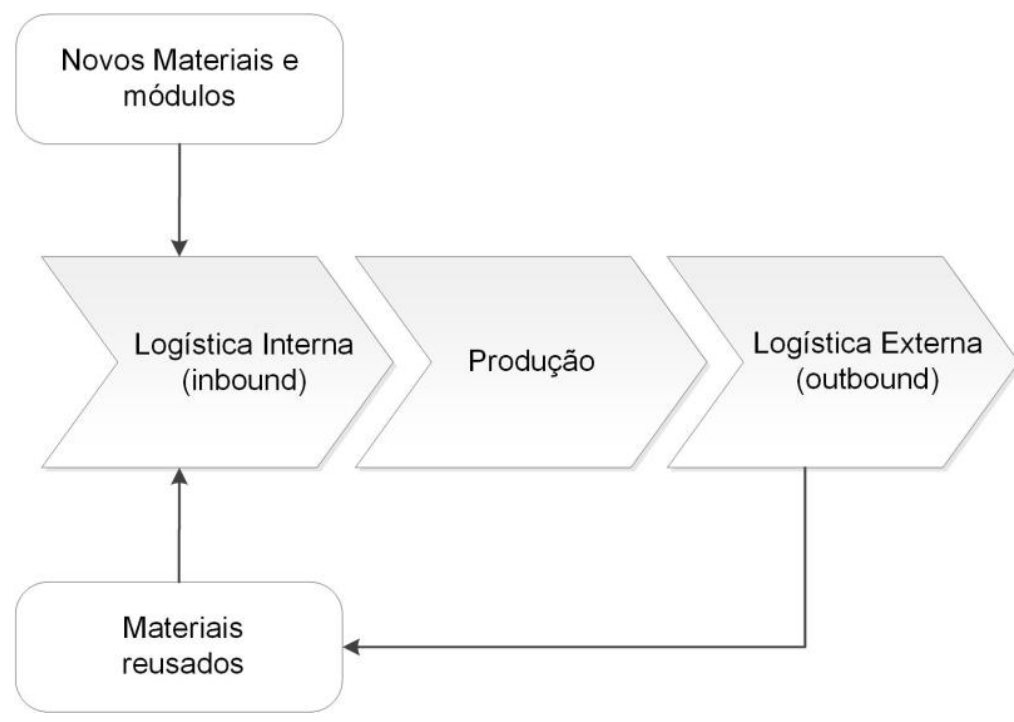

Figura 1. Processo logístico direto e reverso.

Pokharel e Mutha (2009) detalham ainda mais as relações dentro da rede de LR num modelo que contém entradas (inputs), estrutura, processos e saídas (outputs). Os autores incluem como subprocessos da LR a coleta, a inspeção, o processamento, a remanufatura e a consolidação (Figura 2).

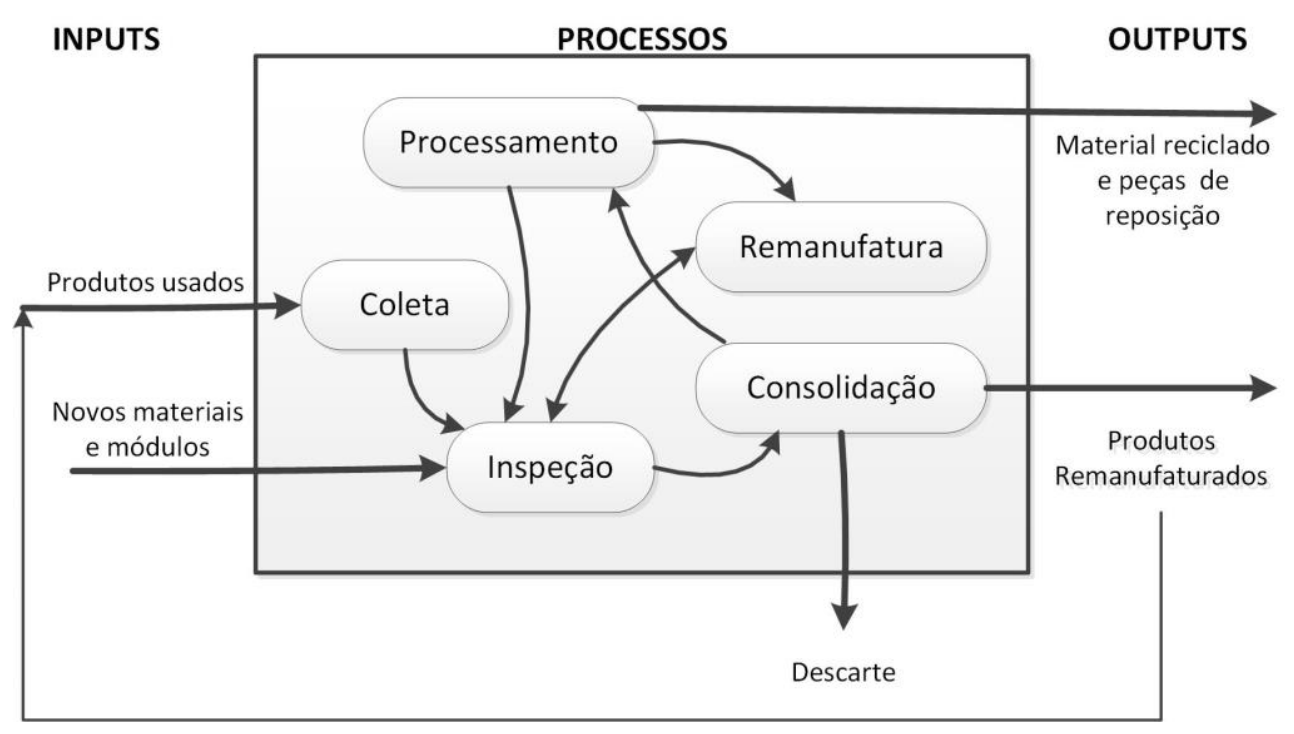

Figura 2. Processo logístico reverso detalhado. Fonte: Pokharel e Mutha (2009).

Como pode se observar na Figura 2, as entradas se referem a produtos ou materiais usados e reciclados, partes usadas como também a novas partes ou módulos que alimentam o processo logístico reverso. Produtos usados passam através de um processo de coleta, por meio de locais de coleta designados ou por meio dos centros de varejo. As partes novas passam por uma inspeção, onde são integrados a produtos usados. No processo de inspeção, tanto produtos novos quanto usados são segregados em diferentes níveis de qualidade para facilitar a decisão de descarte, pequeno reprocessamento ou re-manufatura.

Já o processamento ajuda na desmontagem de peças, algumas das quais podem ser re-usadas e outras encaminhadas para o mercado de peças de reposição. Também, novas peças ou módulos podem 
ser necessárias para a re-manufatura, quando há falta de peças usadas. Por outro lado, as saídas do processo logístico reverso são os produtos, materiais ou peças de reposição recicladas, e os produtos remanufaturados. Por fim, a Figura 2 também apresenta o fluxo se realimentação de materiais, a partir inserção de produtos re-manufaturados como entradas (inputs) na forma de produtos usados, iniciando novamente o ciclo de logística reversa.

Como pode se perceber, existem custos significativos associados com a instalação e operação das redes de LR, por outro lado, essas operações podem representar redução de custos ou receitas potenciais para as manufatureiras (FERGUSON; SOUZA, 2010). Em algumas indústrias, a instalação da rede de LR depende das decisões dos gerentes e diretores das mesmas; já em outras, depende da obrigação a legislações específicas, tais como a Diretiva da União Europeia 2003/108/EC que obriga a coleta de desperdiço elétrico e eletrônico na ordem de 4 quilogramas por habitante por ano, e dependendo da categoria de produto, metas de reuso e reciclagem da ordem de $75 \%$ (em peso).

Seja como cumprimento à legislação, à responsabilidade social ou à obtenção de benefícios financeiros potenciais, um número cada vez maior de empresas começa a adotar ações orientadas a práticas de LR nas suas cadeias de suprimentos (VAZ et al., 2013). Por este motivo, o projeto da cadeia logística reversa começa a ganhar importância, levantando por sua vez, questões importantes (FERGUSON; SOUZA, 2010), desta forma, por exemplo:

- Qual é a estrutura de canais reversos mais adequada? Por exemplo, a manufatureira deve aproveitar sua rede existente de varejo, ou contratar uma terceirizada para coletar os produtos usados, ou deve coletá-los ela mesma dos consumidores finais?

- Qual é a melhor estratégia de coleta? Os produtos usados deveriam ser coletados do usuário final ou é melhor designar locais de entrega para esses produtos? Como influenciam os benefícios financeiros e a estratégia de coleta na estrutura da rede de LR? E fundamentalmente, quais são os impactos da variabilidade da demanda - efeito chicote - na cadeia de suprimentos quando da incorporação de operações de logística reversa?

Neste sentido, quais são as características do efeito chicote? Quais as causas que a provocam? Na seção a seguir, far-se-á uma apresentação sucinta da literatura existente sobre o efeito chicote.

\section{Efeito Chicote - Bullwhip Effect}

O efeito chicote pode ser entendido como a amplificação da demanda ao longo da cadeia de suprimentos (GEARY; DISNEY; TOWILL, 2006). Embora a primeira referência ao termo 'efeito chicote' possa ser rastreada no trabalho de Lee, Padmanabhan e Whang (1997), eles mesmos reconhecem o objeto de pesquisa como sendo o mesmo investigado por J. Forrester no livro 'Industrial Dynamics', a obra seminal da dinâmica de sistemas, metodologia de simulação criada por Forrester para estudar, inicialmente, os ciclos de realimentação de informação e materiais em sistemas de manufatura (FORRESTER, 1961).

Posteriormente, outro dos principais autores da dinâmica de sistemas, J. Sterman, apresenta evidências empírica sobre o efeito chicote, por meio do "jogo da cerveja" ou "beer distribution game" (STERMAN, 1989). Embora, exista um amplo número de trabalhos com foco nos impactos do efeito chicote, é comumente aceito que a instabilidade causada por ele acaba aumentando o custo do fornecimento ao cliente final (LEE; PADMANABHAN; WHANG, 1997; DISNEY; TOWILL, 2003). principais:

Com relação as causas do efeito chicote, Lee, Padmanabhan e Wang (1997) identifica quatro

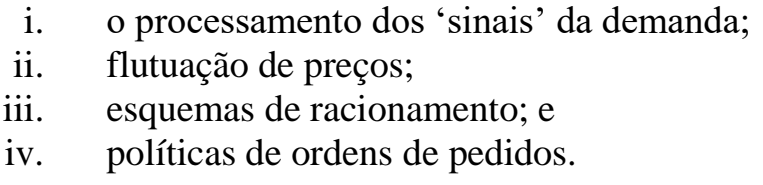

O processamento dos 'sinais' refere-se aos fatores relacionados com a capacidade de efetuar previsões precisas e rápidas, em especial, quando o fluxo produtivo apresenta lead time longos. Os preços, por outro lado, exercem também influência e podem causar o efeito chicote, em especial quando há flutuações de preço importantes, devido a fatores internos (como promoções e vendas com desconto) 
e fatores externos (mudanças na taxa de câmbio, por exemplo). $\mathrm{O}$ terceiro fator considerado, são os esquemas de racionamento, onde as condições da cadeia são desconhecidas, por exemplo, pela falta de compartilhamento de informações entre os atores da cadeia. E por fim, as políticas de ordens de pedidos, que dependem dos métodos para reposição de materiais bem como para reposição de estoque bem como os custos associados de compra.

Por outro lado, existem também consequências tanto locais quanto sistêmicas, para fabricantes, distribuidores e varejistas (FIORIOLLI; FOGLIATTO, 2009). Dentre as causas internas, pode-se destacar os baixos níveis de serviço, vendas perdidas, aumento do estoque de segurança e gestão ineficiente dos recursos. Já, para as causas externas: elevação dos custos relacionados a estoques, queda do retorno sobre o capital investido, queda da produtividade dos funcionários, processo decisórios reativos, e a gestão ineficiente da cadeia como um todo.

Pode-se observar que os esforços de pesquisa têm se centrado em atenuar/eliminar a propagação do efeito chicote ao longo da cadeia. Assim, estudos que apliquem modelos quantitativos têm aumentado ao longo dos anos, com o objetivo de entender as causas da propagação e, principalmente, de eliminálas, por meio da simulação e outros modelos analíticos (FIORIOLLI; FOGLIATTO, 2009). Assim, as abordagens utilizadas para a análise quantitativa do efeito chicote podem ser dividas em três tipos: i) os estudos que utilizam o cálculo da relação entre a variância de pedidos e variância da demanda (LEE; PADMANABHAN; WHANG, 1997; CHEN et al., 2000; FIORIOLLI; FOGLIATTO, 2009); ii) cálculo da relação entre a taxa de pedidos e a taxa da demanda (WARBURTON, 2004); e iii) cálculo do quociente entre o coeficiente de variação da demanda gerada por um nível da cadeia e o coeficiente de variação da demanda recebida por este mesmo nível (FRANSOO; WOUTERS, 2000).

Este estudo, embora não tenha como objetivo avaliar o efeito chicote em si, e sim, avaliar a diferença entre os efeitos chicote de cadeias sem e com logística reversa, inspira-se na avaliação da variação entre as taxas de pedidos e a de demanda, conforme apresentado acima.

\section{Modelagem de Cadeias Logísticas Reversas}

Embora exista um volume de literatura amplo e crescente sobre LR, as ferramentas de análise dessas redes não têm sido completamente exploradas (ROGERS, DALE S; MELAMED; LEMBKE, 2012).

Desta forma, Fleischmann et. al. (1997) são os primeiros em oferecer uma atenção especial para as ferramentas de modelagem que podem ajudar a planejar e gerenciar as complexas estruturas das cadeias logísticas reversas, embora o estudo dos autores esteja mais relacionado com descrever as diferentes configurações de LR e não em oferecer ferramentas analíticas ou de simulação per se.

Mais recentemente, Rogers, Melamed e Tibben-Lembke (2012) discutem ambas abordagens de modelagem (analíticas e de simulação) como formas possíveis de estudar o comportamento das redes de LR. Os autores argumentam que os modelos analíticos incluem um conjunto de equações e restrições que são utilizáveis unicamente quando o modelo é relativamente simples, fazendo difícil, portanto, a modelagem analítica de processos mais complexos de LR. Por outro lado, os autores argumentam que a simulação de modelos pode oferecer certas vantagens para capturar a estrutura de qualquer rede de LR em algoritmos computacionais.

Como descrito anteriormente, a Dinâmica de Sistemas tem sido utilizada em várias aplicações e estudos relacionados com cadeias de suprimentos (ANGERHOFER; ANGELIDES, 2000). Mais recentemente, Das e Dutta utilizaram DS para analisar uma política de logistica reversa de coleta de materiais (DAS; DUTTA, 2013); por outro lado, Mafakheri e Nasiri utilizaram DS para formular as regras de decisão entre fabricante e varejista na escolha das receitas ótimas, numa cadeia logística reversa (MAFAKHERI; NASIRI, 2013). Uma revisão mais ampla de aplicações de dinâmica de sistemas na logística reversa pode ser encontrada em Vaz, Inomata e Uriona (VAZ; INOMATA; URIONA, 2016)

Neste sentido, a Dinâmica de Sistemas pode ser uma potencial ferramenta de explicação das relações complexas e não-lineares formadas nas redes de LR, as quais são diferentes daquelas da logística direta, conforme citam Rogers, Melamed e Tibben-Lembke (2012) quando explicam a crença 
errada de que a logística reversa é exatamente igual que a logística direta, e que consiste apenas de dirigir os caminhões na direção oposta.

\section{Resultados e Discussão}

Nesta seção apresentamos um exemplo de aplicação de DS para a modelagem e simulação de uma cadeia logística reversa, ressaltando dessa maneira os possíveis impactos de amplificação (efeito chicote) na cadeia de suprimentos, quando da implantação de uma rede de logística reversa. Para isto, iremos analisar uma cadeia de suprimentos de três níveis (fornecedor-produtor-distribuidor), usualmente encontrada no setor eletroeletrônico (fabricação de aparelhos e equipamentos elétricos e eletrônicos). Para a calibração do modelo utilizaram-se dados de uma empresa deste setor, localizada em Florianópolis, no Sul do Brasil, com vinte anos no mercado.

O modelo representa, essencialmente, a estrutura física (fluxos materiais) da cadeia bem como as principais regras de decisão utilizadas para a gestão da mesma. Para isto, iremos apresentar primeiramente a estrutura da cadeia de suprimentos sem levar em consideração os processos de logística reversa, ou seja, a cadeia logística direta. A Figura 3 mostra um esquema da macroestrutura do modelo antes da implantação da rede de logística reversa.

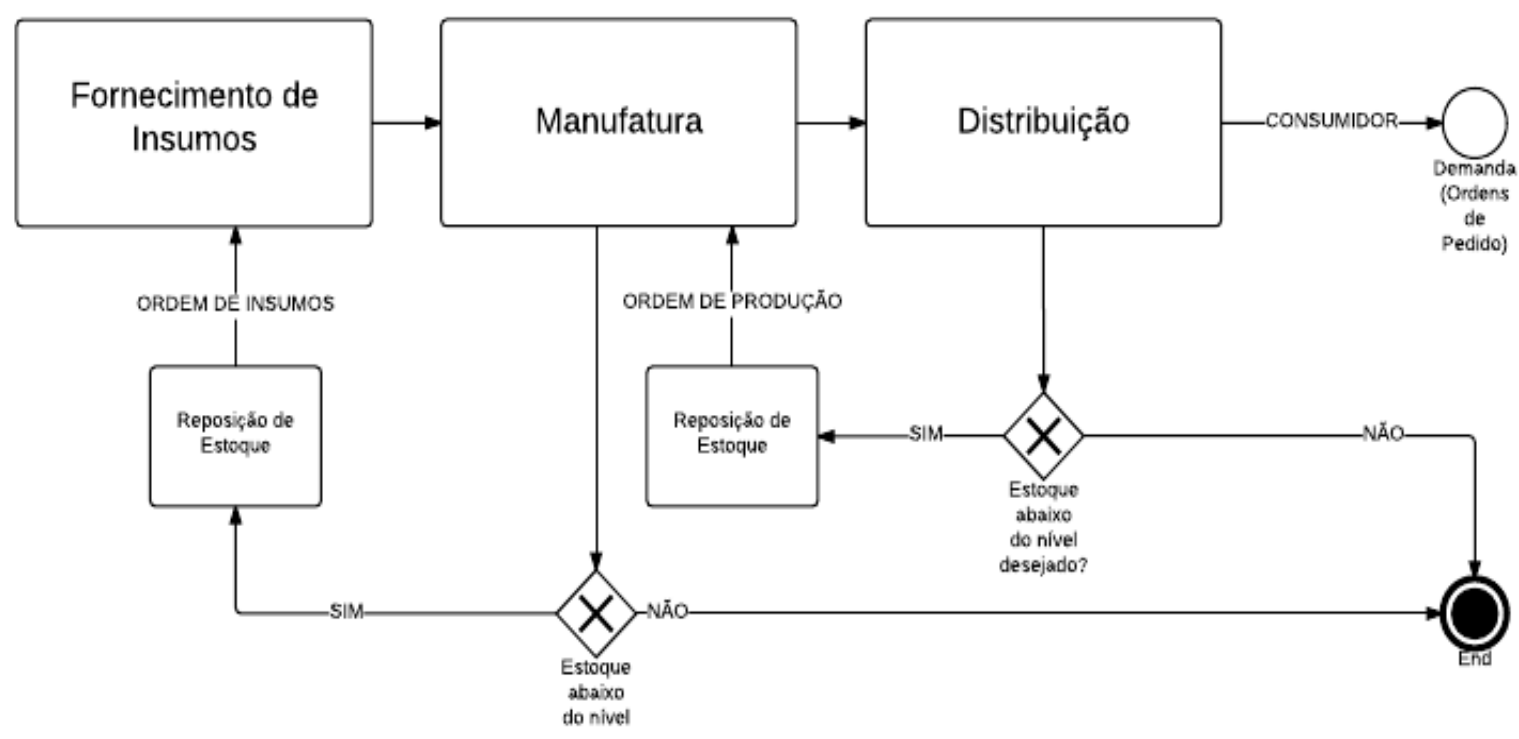

Figura 3. Esquema da cadeia antes da implantação da rede de logística reversa

\subsection{Modelo01: Cadeia de Suprimentos 'Forward'}

Conforme a Figura 3, o modelo apresenta três elos da cadeia, onde o fluxo de materiais segue um único sentido 'forward', este modelo será denominado Modelo01. Por outro lado, as atividades de reposição de estoque respondem a fluxos de informação, a partir da previsão de demanda e das necessidades de reposição de estoque. O Modelo01 foi desenvolvido a partir da proposta de Sterman (STERMAN, 2000) e na Figura 4 é apresentada uma versão simplificada para facilitar a compreensão do mesmo.

Para demonstrar o efeito chicote produzido unicamente pelo fluxo 'forward' na cadeia, assumimos que o comportamento das ordens de pedidos do consumidor (demanda) segue uma tendência senoidal, para um período total de simulação de 50 semanas $^{1}$. Conforme observado na Figura 5 (gráfico superior), a media de pedidos é de 10.000 unidades do produto. A Figura 5 (gráfico superior) apresenta

\footnotetext{
${ }^{1}$ A tendência senoidal servirá para representar, principalmente, o comportamento de sazonalidade dos produtos da empresa. De qualquer forma, para fins práticos, tanto o modelo01 como o modelo02 podem ser facilmente adaptados para outros comportamentos da demanda.
} 
também o atraso de fase (duas semanas aproximadamente) entre as ordens de pedidos reais (linha azul) e a taxa de expedição (linha vermelha). 


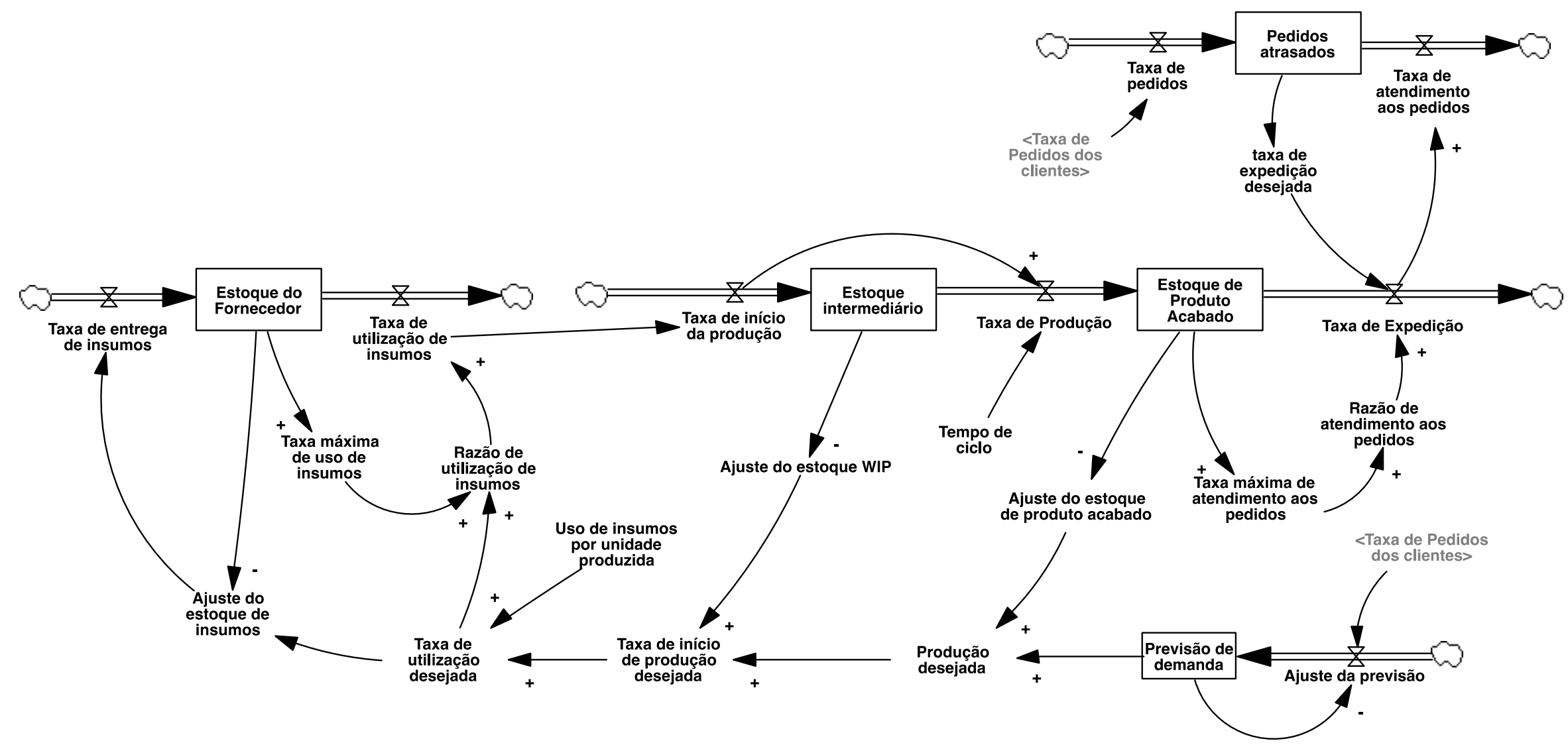

Figura 4. Modelo01: Modelo simplificado da cadeia de suprimentos 'forward'. Fonte: Sterman (STERMAN, 2000) 
Esse atraso relativamente pequeno entre a demanda do consumidor e as entregas no entanto, produz amplificações maiores de demanda nos elos posteriores, conhecido como o 'efeito chicote'. A Figura 5, apresenta também, a amplificação produzida pelo atraso de fase para toda a cadeia 'forward', ou seja, o efeito chicote (gráfico do meio).

Na Figura 5 (gráfico do meio) é possível observar a amplificação da demanda quando incluídos os gráficos do início da produção (linha verde) e da taxa de entrega dos insumos por parte do fornecedor (linha amarela). Em ambos processos observa-se uma amplificação maior do que aquela observada na taxa de expedição do produtor. Observa-se também que a taxa de pedidos (linha azul) possui aproximadamente a mesma fase do que a entrega de insumos por parte do fornecedor (linha amarela), ou seja, não existe defasagem entre ambas taxas, porém existe um alto nível de amplificação. Como a produção está mais próxima do consumidor, seguindo a teoria do efeito chicote, possui uma amplificação menor à taxa de início da produção, porém, a produção não é instantânea, ela precisa de 8 semanas (de acordo com os parâmetros do modelo) para produzir um novo lote de produção, o que explica a defasagem entre a taxa de início da produção (linhas verde) e taxa de produção (linha preta).

Por fim, a Figura 5 (gráfico inferior) apresenta o comportamento dos diferentes estoques ao longo da cadeia. Observa-se que o estoque WIP (produto em processo) apresenta uma flutuação considerável (de 60.000 a 100.000 unidades ou peças) em relação ao estoque de produto acabado, que apresenta uma tendência de flutuação mais suave. Isto se deve, principalmente ao estoque de produto em processo depender de duas informações críticas e a seus atrasos inerentes: a reposição de estoque, a partir das informações das ordens de pedido; e a flutuação de entregas dos fornecedores. 

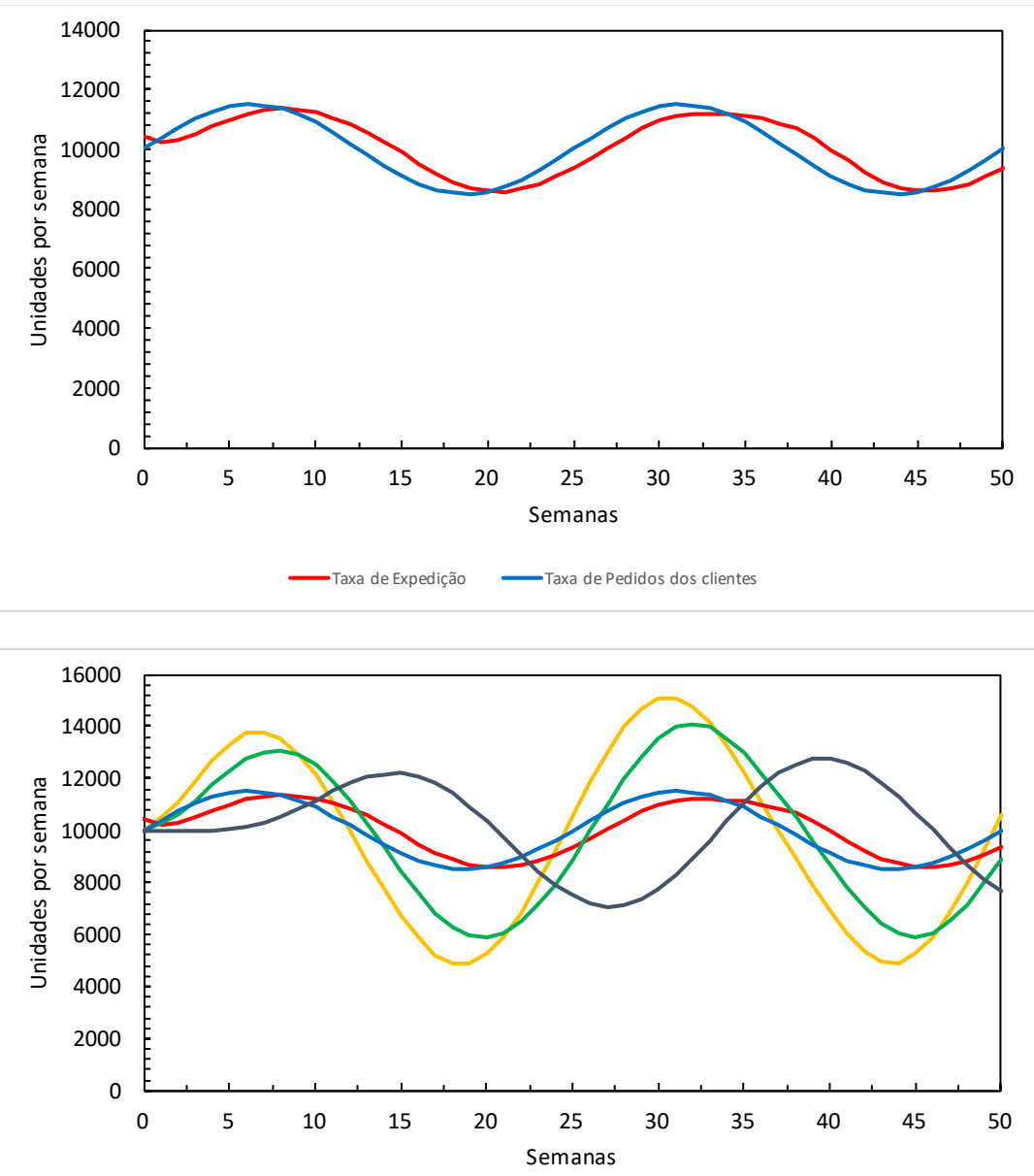

- Taxa de entrega de insumos — Taxa de Expedição

—Taxa de início da produção

—Taxa de Pedidos dos clientes — Taxa de Produção

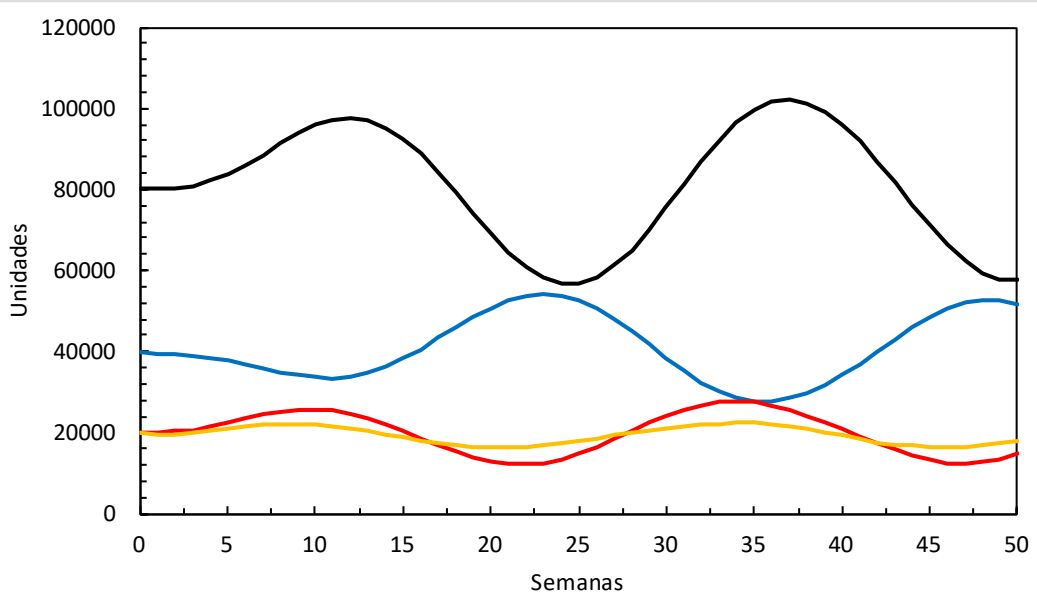

- Estoque de Produto Acabado - Estoque do Fornecedor - Estoque WIP —Pedidos atrasados

Figura 5. Amplificação da demanda (efeito chicote) na cadeia 'forward' 
Conforme a Figura 6, o modelo apresenta os três elos da cadeia, onde o fluxo de materiais segue dois sentidos, o sentido da produção-consumo (forward) e o sentido reverso (do consumo à produção) em linha pontilhada, este modelo será denominado Modelo02.

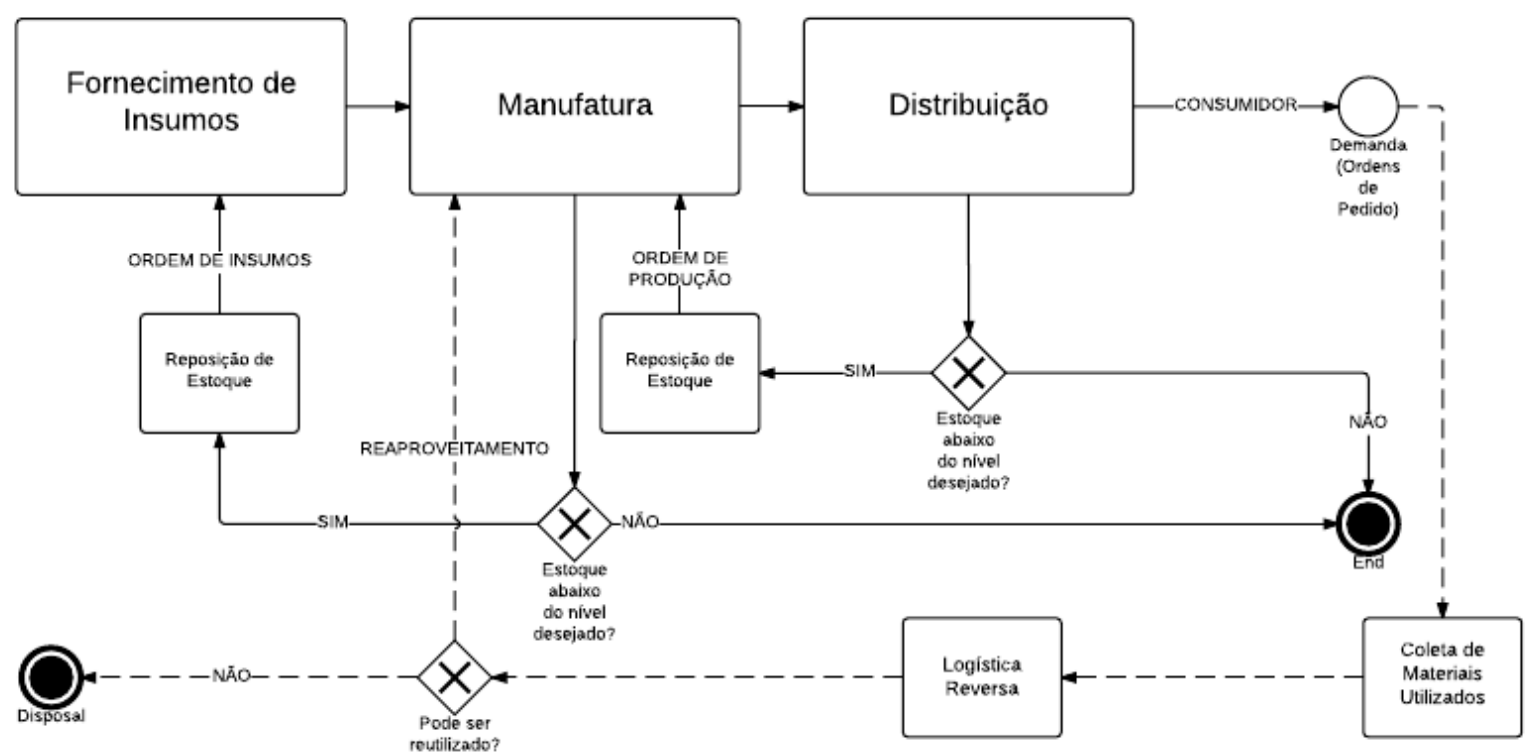

Figura 6. Esquema da cadeia após a implantação da rede de logística reversa

Conforme a Figura 6, as atividades de reposição de estoque respondem a fluxos de informação, a partir da previsão de demanda e à inclusão de materiais re-manufaturados ao processo produtivo. $\mathrm{O}$ Modelo02 simplificado, incluindo a estrutura da cadeia logística reversa, é apresentado na Figura 7. 


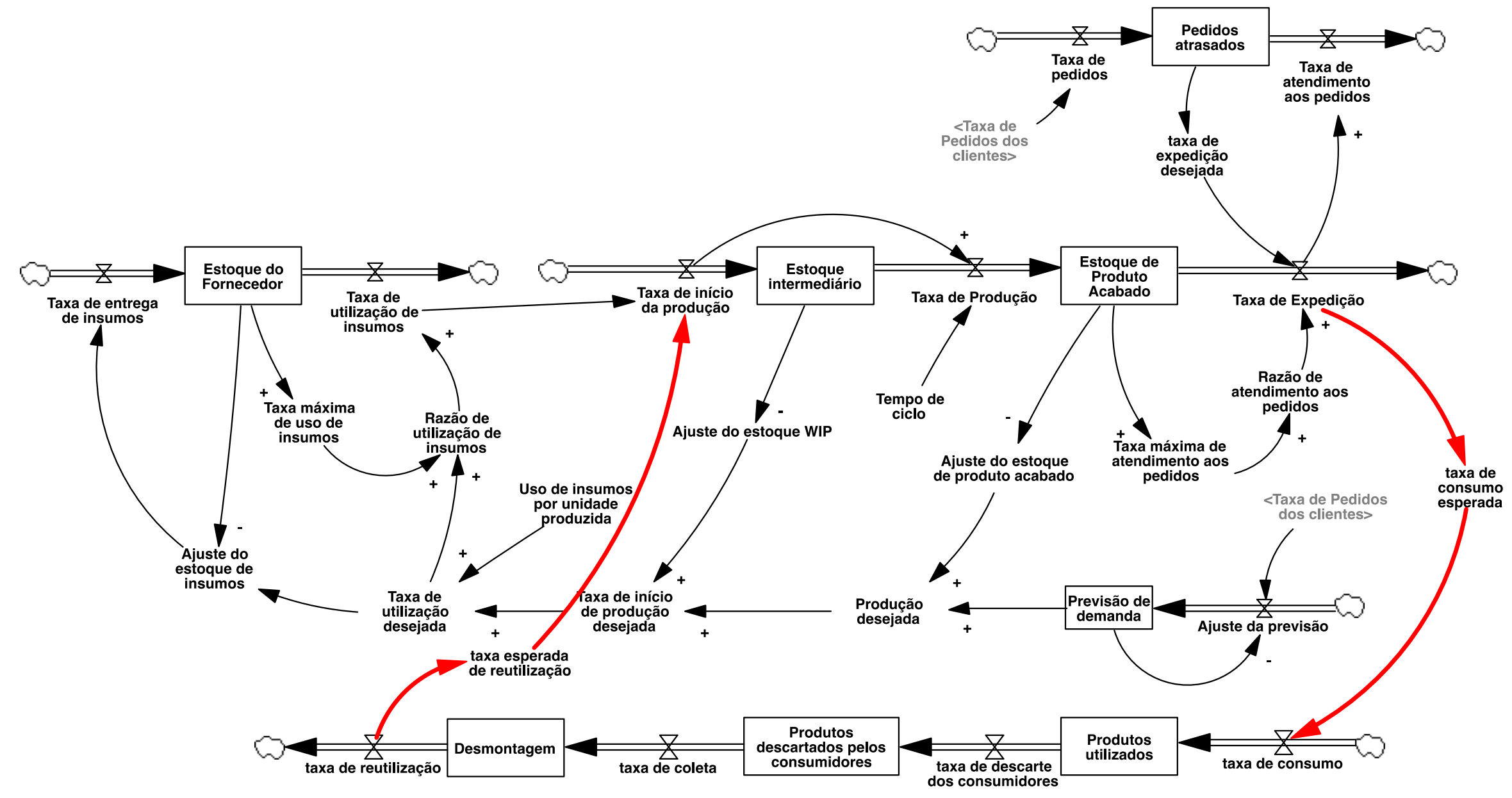

Figura 7. Modelo02: Modelo simplificado da cadeia de suprimentos incluindo logística 'forward' e logística reversa 
Conforme a Figura 7, o Modelo02 inclui a estrutura da logística reversa. As setas em vermelho representam a incorporação da estrutura de logística reversa ao modelo previamente apresentado. A seguir, apresenta-se na Figura 8 o efeito chicote amplificado a partir da inclusão das operações de logística reversa.
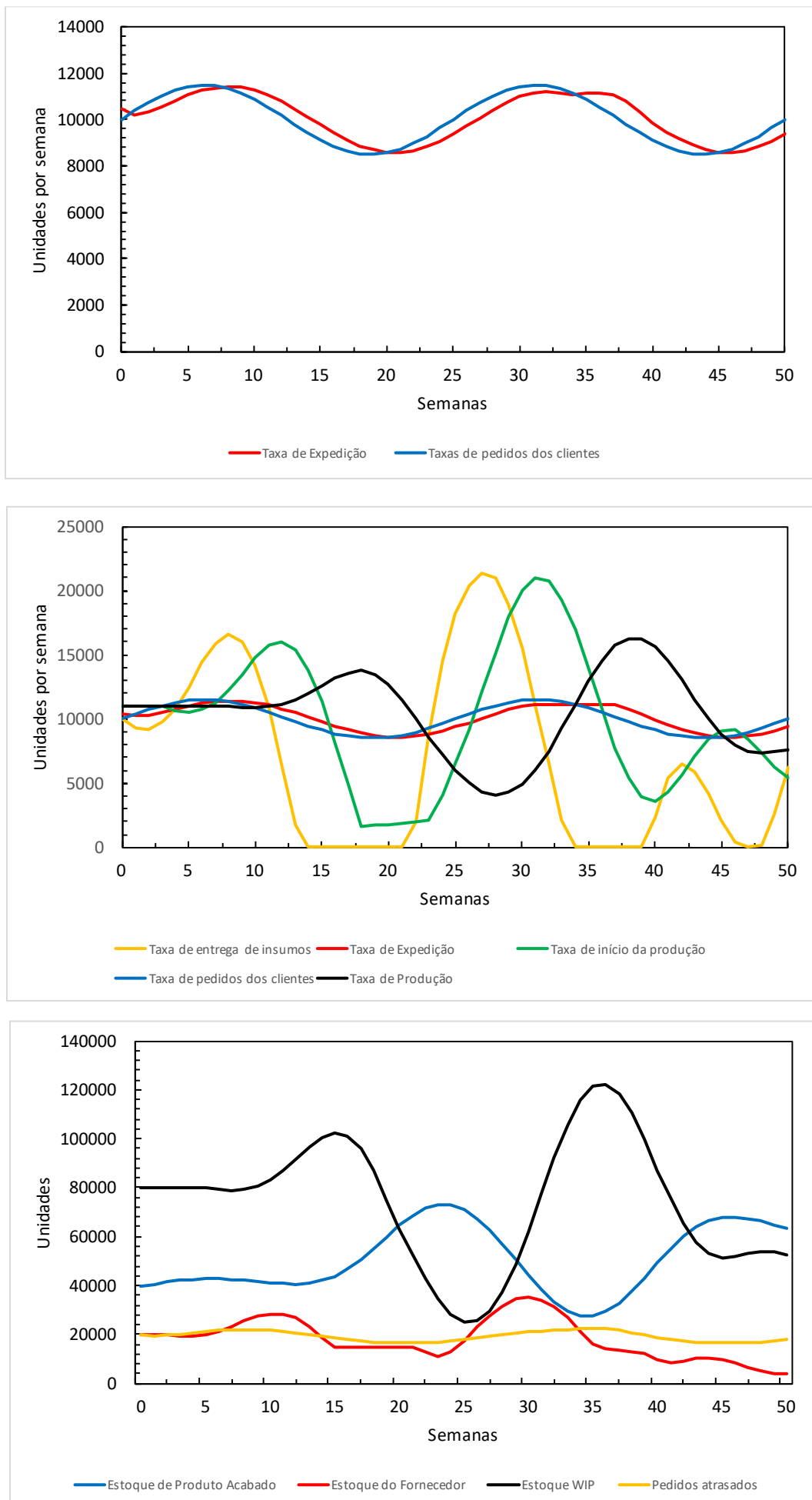

Figura 8. Amplificação da demanda (efeito chicote) na cadeia com logística 'forward' e logística reversa. 
Ao compararmos a Figura 8 (com logística reversa) com a Figura 5 (sem logística reversa) identifica-se uma amplificação da demanda muito maior quando a logística reversa é incorporada nas operações da empresa. Embora a taxa de expedição (linha vermelha nas Figs. 5 e 8) não tenha sido afetada significativamente pela inclusão de operações de logística reversa, o efeito de amplificação é observado nos outros elos da cadeia: no início da produção (linha verde nas Figs. 5 e 8), na taxa de entrega dos insumos por parte do fornecedor (linha amarela nas Figs. 5 e 8) e na taxa de produção (linha preta nas Figs. 5 e 8).

Com relação a taxa de entrega de insumos, vale a pena salientar que refere-se a recebimentos de insumos (materiais) que são solicitados a partir das necessidades de materiais pelo processo produtivo. Quando, por exemplo, na semana 15, essa taxa é igual a zero (Figura 8-meio), o sistema absorve todo o material requerido a partir do reuso de materiais que foram incorporados ao processo produtivo a partir das operações da cadeia reversa. Este comportamento observa-se novamente na semana 39 até a semana 44.

Para verificar com mais detalhe as diferenças de amplificação entre o cenário sem logística reversa e o cenário com logística reversa, apresentam-se a seguir gráficos comparativos entre os dois cenários, começando pela taxa de expedição que é aquela mais próxima da demanda real e continuando para trás, até o elo mais afastado da cadeia, a taxa de entrega de insumos. A figura 9 apresenta os gráficos comparativos em questão, apresentando a variação percentual por semana, para o período total de 50 semanas simuladas.
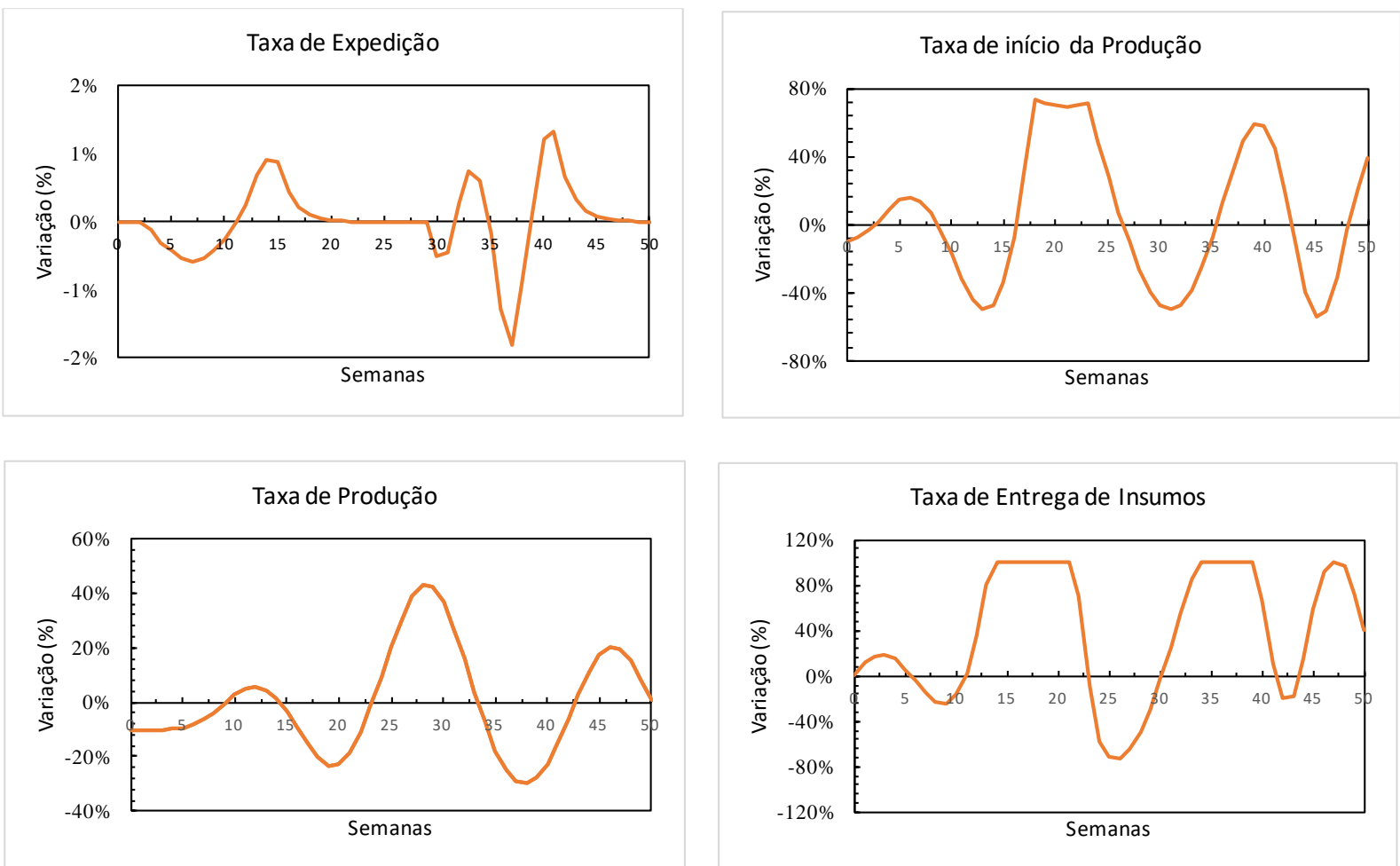

Figura 9. Variações percentuais entre os modelos 'forward' e 'forward + reverse'.

Como observado nas Figuras 9 existe uma diferença de fase entre o cenário onde a empresa não conta com operações de logística reversa e o cenário onde essas operações são incorporadas. Também é possível observar que o efeito chicote é amplificado à medida que os elos da cadeia ficam mais afastados da demanda real. Iniciando pela 'taxa de expedição', pode-se observar variações pequenas entre ambas cadeias, chegando no valor máximo de amplificação de aproximadamente $-2 \%$, ou seja, a cadeia 'forward + reverse' conseguiu entregar $2 \%$ a menos de produtos para os clientes na pior semana, dentre as 50 simuladas.

Já, na 'taxa de início de produção' observa-se um incremento da variação entre ambas cadeias, chegando a uma diferença de quase $+80 \%$ na pior semana simulada. Ou seja, a cadeia 'forward + 
reverse' iniciou a produção de um $80 \%$ a mais, com relação a cadeia 'forward'. No entanto, outras semanas também apresentaram variações significativas, embora não tão altas como a dos $+80 \%$, o que significa que o efeito chicote se agravou quando da incorporação das operações de logística reversa. A defasagem entre ambas as cadeias pode ser explicada a partir dos tempos adicionais incorporados para o processamento dos materiais e insumos usados e da decomposição dos mesmos antes de entrar no processo produtivo.

Um comportamento similar pode ser observado na 'taxa de produção', com um incremento da variação à medida que a simulação avança, chegando a um valor máximo de aproximadamente $+40 \%$ de variação entre ambas as cadeias. No entanto, no elo onde existe maior diferença de amplificação e de fase entre ambos os cenários, é a 'taxa de entrega de insumos' onde são observadas variações de até $+100 \%$ e $-80 \%$ ao longo das semanas.

\section{Conclusões}

Observa-se um interesse crescente por incorporar operações de logística reversa em diversos setores, motivados pelas pressões da legislação, dos concorrentes e mercado e pelos próprios consumidores. Embora, no Brasil, não existam legislações de ampla abrangência nos diversos setores industriais, observa-se também, uma tendência internacional de formulação de políticas e regulamentos, que virão a ser implantados no país, mais cedo do que tarde.

O artigo apresentou, por meio da simulação de dinâmica de sistemas, um estudo sobre os impactos que a implementação de cadeias de logística reversa podem ter no efeito chicote, e portanto, na eficiência, lucratividade e desempenho das empresas industriais. Para isto, os modelos construídos possibilitam visualizar os efeitos produzidos pela incorporação de fluxos de materiais reversos, principalmente a amplificação do efeito chicote e a formação de oscilações de menor amplitude ao longo do tempo de operação da cadeia logística, a partir dos dados de uma empresa do setor eletroeletrônico.

Embora os modelos estejam num nível altamente agregado, foi possível observar que a implantação de operações de logística reversa afetam e agravam o efeito chicote na empresa. Esta conclusão pode ser, de forma geral, ampliada para outros tipos de empresas de diversos setores, pelo fato do modelo se encontrar, justamente, num nível alto de agregação. Foi possível confirmar também a amplificação da variação para cada elo da cadeia, concluindo que, a medida que os elos se afastam do consumidor final, essa variação é amplificada, chegando no elo do fornecedor, com variações de até $+100 \%$ e $-80 \%$ no número de pedidos de insumos, gerando uma espécie de efeito chicote da própria implantação da logística reversa.

Esses resultados possibilitam oferecer à empresa em estudo, uma ferramenta que ajude na implantação da cadeia de logística reversa, tentando, a partir da geração de cenários no modelo, atenuar os impactos dessa implantação. Principalmente, o modelo possibilita visualizar os impactos que a cadeia de logística reversa trará em termos do efeito chicote.

Por fim, esse tipo de modelos de simulação possibilita, portanto, analisar as características de funcionamento da cadeia logística direta e reversa e apresenta-se como uma ferramenta com alto potencial para a formulação de estratégias e para a tomada de decisões de gestores bem como de pesquisadores interessados em cadeias de suprimentos, e em particular, em operações de logística reversa.

\section{Referências}

ANGERHOFER, B. J.; ANGELIDES, M. C. System dynamics modelling in supply chain management: research review. Simulation Conference, 2000. Proceedings. Winter, 2000. 2000. p.342-351 vol.1.

BORSHCHEV, A.; FILIPPOV, A. From System Dynamics and Discrete Event to Practical Agent Based Modeling : Reasons, Techniques, Tools. Proceedings of the 22nd International Conference of the System Dynamics Society. Oxford, England: The System Dynamics Society 2004 
CARTER, C. R.; ELLRAM, L. M. Reverse Logistics: A Review of the Literature and Framework for Future Investigation. Journal of Business Logistics, v. 19, n. 1, p. 85-102, 1998.

CHEN, F. et al. Quantifying the Bullwhip Effect in a Simple Supply Chain: The Impact of Forecasting, Lead Times, and Information. Management Science, v. 46, n. 3, p. 436-443, 2000.

DAS, D.; DUTTA, P. A system dynamics framework for integrated reverse supply chain with three way recovery and product exchange policy. Computers \& Industrial Engineering, v. 66, n. 4, p. 720-733, 2013.

DISNEY, S. M.; TOWILL, D. R. The effect of vendor managed inventory (VMI) dynamics on the Bullwhip Effect in supply chains. International Journal of Production Economics, v. 85, n. 2, p. 199$215,8 / 11 / 2003$.

FERGUSON, M. E.; SOUZA, G. C. Closed-loop supply chains: new developments to improve the sustainability of business practices. CRC Press, 2010.

FIORIOLLI, J. C.; FOGLIATTO, F. S. Modelagem do Efeito Chicote em ambientes com demanda e lead time estocásticos mediante uma nova política de tratamento dos excessos de estoque. Production, v. 19, p. 27-40, 2009.

FLEISCHMANN, M. et al. Quantitative models for reverse logistics: a review. European journal of operational research, v. 103, n. 1, p. 1-17, 1997.

FORRESTER, J. W. Industrial Dynamics. Cambridge MA: Productivity Press, 1961.

FRANSOO, J. C.; WOUTERS, M. J. F. Measuring the bullwhip effect in the supply chain. Supply Chain Management: An International Journal, v. 5, n. 2, p. 78-89, 2000/05 2000.

GEARY, S.; DISNEY, S. M.; TOWILL, D. R. On bullwhip in supply chains-historical review, present practice and expected future impact. International Journal of Production Economics, v. 101, n. 1, p. $2-18,2006$.

LEE, H. L.; PADMANABHAN, V.; WHANG, S. Information Distortion in a Supply Chain: The Bullwhip Effect. Management Science, v. 43, n. 4, p. 546-558, 1997.

MAFAKHERI, F.; NASIRI, F. Revenue sharing coordination in reverse logistics. Journal of Cleaner Production, v. 59, p. 185-196, 2013.

POKHAREL, S.; MUTHA, A. Perspectives in reverse logistics: a review. Resources, Conservation and Recycling, v. 53, n. 4, p. 175-182, 2009.

ROGERS, D. S.; MELAMED, B.; LEMBKE, R. S. Modeling and analysis of reverse logistics. Journal of Business Logistics, v. 33, n. 2, p. 107-117, 2012.

ROGERS, D. S.; TIBBEN-LEMBKE, R. S. Going backwards: reverse logistics trends and practices. Reverse Logistics Executive Council Pittsburgh, PA, 1999.

SPENGLER, T.; SCHROETER, M. Strategic Management of Spare Parts in Closed-Loop Supply Chains: A system dynamics approach. Interfaces, v. 33, n. 6, p. 7-17, 2003.

STERMAN, J. D. Modeling managerial behavior: Misperceptions of feedback in a dynamic decision making experiment. Management science, v. 35, n. 3, p. 321-339, 1989.

Business Dynamics. Systems Thinking and Modeling for a complex world. Boston: Mc Graw Hill Higher Education, 2000. 1008p. 
STOCK, J. R. Reverse logistics. Council of Logistics Management, 1992.

STOCK, J. R. Development and implementation of reverse logistics programs. ANNUAL CONFERENCE PROCEEDINGS, COUNCIL OF LOGISTICS MANAGEMENT.--, 1998.

VAZ, C. R. et al. Some reasons to implement reverse logistics in companies. International Journal of Environmental Technology and Management, v. 16, n. 5, p. 467-479, 2013.

VAZ, C. R.; INOMATA, D.; URIONA, M. Aplicações de Dinâmica de Sistemas na Logística Reversa: Uma análise bibliométrica. Revista GEPROS, v. 11, n. 4, p. 101-116, 2016.

VLACHOS, D.; GEORGIADIS, P.; IAKOVOU, E. A system dynamics model for dynamic capacity planning of remanufacturing in closed-loop supply chains. Computers \& Operations Research, v. 34, n. 2, p. 367-394, 2007.

WARBURTON, R. D. H. An Analytical Investigation of the Bullwhip Effect. Production and Operations Management, v. 13, n. 2, p. 150-160, 2004. 